\title{
A NOTE ON COMPREHENSIVE BACKWARD BIORTHOGONALIZATION
}

\author{
DAVID K. RUCH
}

Received 16 February 2006; Revised 21 July 2006; Accepted 25 July 2006

We present a backward biorthogonalization technique for giving an orthogonal projection of a biorthogonal expansion onto a smaller subspace, reducing the dimension of the initial space by dropping $d$ basis functions. We also determine which basis functions should be dropped to minimize the $L^{2}$ distance between a given function and its projection. This generalizes some recent results of Rebollo-Neira.

Copyright (c) 2006 Hindawi Publishing Corporation. All rights reserved.

In [3], Rebollo-Neira gives a backward biorthogonalization technique for projecting a biorthogonal expansion onto a subspace, reducing the dimension $N$ of the initial space by dropping $d=1$ basis function. In this note, we generalize this method to reduce the space by an arbitrary number $d$ of basis functions, $d<N$. Proposition 3.4 in [3] indicates which single basis function is to be removed in order to minimize the $L^{2}$ distance between a function $f$ and its orthogonal projection into the reduced space. We will also generalize this result in Proposition 7. If more than one basis function is to be dropped, Rebollo-Neira recommends iterating the $d=1$ process. We show via Example 8 that in some circumstances iterating the $d=1$ process $k$ times leads to results inferior to using Proposition 7 and dropping $k=d$ basis functions simultaneously.

We begin with a Hilbert space $H$ and an $N$-dimensional subspace $V$. Assume biorthogonal bases of $V$ given by $\left\{x_{i}^{\prime}\right\}_{i=1}^{N}$ and $\left\{x_{i}\right\}_{i=1}^{N}$ such that $\left\langle x_{i}^{\prime}, x_{j}\right\rangle=\delta_{i j}$. Now drop $d$ basis elements from each set, without loss of generality the first $d$ elements for notational purposes, and form the reduced subspaces $\widetilde{V}=\operatorname{span}\left\{x_{i}\right\}_{i=d+1}^{N}$ and $\tilde{V}^{\prime}=\operatorname{span}\left\{x_{i}^{\prime}\right\}_{i=1}^{d}$. We wish to modify the $x_{i}^{\prime}$ so that the projection from $V$ to $\tilde{V}$ is orthogonal. We next recursively construct the sequence $\left\{v_{i}^{\prime}\right\}_{i=1}^{d} \subset \tilde{V}^{\prime}$ by

$$
v_{1}^{\prime}=x_{1}^{\prime}, \quad v_{i}^{\prime}=x_{i}^{\prime}-\sum_{\ell=1}^{i-1} \frac{\left\langle x_{i}^{\prime}, v_{\ell}^{\prime}\right\rangle}{\left\langle v_{\ell}^{\prime}, v_{\ell}^{\prime}\right\rangle} v_{\ell}^{\prime}, \quad i \leq d .
$$

Hindawi Publishing Corporation International Journal of Mathematics and Mathematical Sciences Volume 2006, Article ID 87392, Pages 1-5

DOI 10.1155/IJMMS/2006/87392 
2 A note on comprehensive backward biorthogonalization

We observe that the set $\left\{v_{i}^{\prime}\right\}_{i=1}^{d}$ forms an orthogonal basis of $\tilde{V}^{\prime}$ by construction. We then construct the sequence $\left\{\tilde{x}_{i}^{\prime}\right\}_{i=d+1}^{N}$ by

$$
\tilde{x}_{i}^{\prime}=x_{i}^{\prime}-\sum_{\ell=1}^{d} \frac{\left\langle x_{i}^{\prime}, v_{\ell}^{\prime}\right\rangle}{\left\langle v_{\ell}^{\prime}, v_{\ell}^{\prime}\right\rangle} v_{\ell}^{\prime}
$$

and set $U=\operatorname{span}\left\{\tilde{x}_{i}^{\prime}\right\}_{i=d+1}^{N}$. We will see that this formula generalizes the dual modification of [3, Theorem 3.1] for $d \geq 1$. Note that each $\tilde{x}_{i}^{\prime}$ is created to be orthogonal to $\tilde{V}^{\prime}$ by subtracting from $x_{i}^{\prime}$ its projection onto $\tilde{V}^{\prime}$.

Proposition 1. The spaces $U$ and $\tilde{V}^{\prime}$ are orthogonal complements in $V, V=\tilde{V} \oplus \tilde{V}^{\prime}$.

Proof. Choose $i, j$ such that $j \leq d<i$ and use the definition of $\tilde{x}_{i}^{\prime}$ and the orthogonality of $\left\{v_{i}^{\prime}\right\}$,

$$
\left\langle\tilde{x}_{i}^{\prime}, v_{j}^{\prime}\right\rangle=\left\langle x_{i}^{\prime}, v_{j}^{\prime}\right\rangle-\sum_{\ell=1}^{d} \frac{\left\langle x_{i}^{\prime}, v_{\ell}^{\prime}\right\rangle}{\left\langle v_{\ell}^{\prime}, v_{\ell}^{\prime}\right\rangle}\left\langle v_{\ell}^{\prime}, v_{j}^{\prime}\right\rangle=\left\langle x_{i}^{\prime}, v_{j}^{\prime}\right\rangle-\left\langle x_{i}^{\prime}, v_{j}^{\prime}\right\rangle=0 .
$$

Thus $U$ and $\tilde{V}^{\prime}$ are orthogonal subspaces of $V$, and their dimensions add to $N$.

We next verify that $U$ and $\tilde{V}$ are actually the same space.

Lemma 2. The spaces $U$ and $\tilde{V}^{\prime}$ are orthogonal complements in $V$, and $U=\tilde{V}$.

Proof. By (1), we can write $v_{j}^{\prime}=\sum_{n=1}^{j} a_{n} x_{n}^{\prime}$ for some constants $a_{n}$, so the original biorthogonality condition $\left\langle x_{i}^{\prime}, x_{j}\right\rangle=\delta_{i j}$ says that, for $j\left\langle i,\left\langle v_{j}^{\prime}, x_{i}\right\rangle=\sum_{n=1}^{j} a_{n}\left\langle x_{n}^{\prime}, x_{i}\right\rangle=0\right.$. Thus $\tilde{V}$ and $\tilde{V}^{\prime}$ are orthogonal subspaces of $V$, and their dimensions add to $N$. By the previous proposition, $U=\tilde{V}$.

Next we give the desired biorthogonal bases of the reduced subspace $\tilde{V}$.

Proposition 3. The reduced spaces $U$ and $\tilde{V}$ are identical and have biorthogonal bases $\left\{\tilde{x}_{i}^{\prime}\right\}_{i=d+1}^{N}$ and $\left\{x_{j}\right\}_{j=d+1}^{N}$.

Proof. Using Lemma 2 and (2), we have for $i, j>d \geq \ell$,

$$
\left\langle\tilde{x}_{i}^{\prime}, x_{j}\right\rangle=\left\langle x_{i}^{\prime}, x_{j}\right\rangle-\sum_{\ell=1}^{d} \frac{\left\langle x_{i}^{\prime}, v_{\ell}^{\prime}\right\rangle}{\left\langle v_{\ell}^{\prime}, v_{\ell}^{\prime}\right\rangle}\left\langle v_{\ell}^{\prime}, x_{j}\right\rangle=\delta_{i j}-\sum_{\ell=1}^{d} \frac{\left\langle x_{i}^{\prime}, v_{\ell}^{\prime}\right\rangle}{\left\langle v_{\ell}^{\prime}, v_{\ell}^{\prime}\right\rangle} \cdot 0=\delta_{i j} .
$$

In order to give an explicit method for determining which basis functions to drop to minimize the residual, we give a formula for the projection operator.

Proposition 4. The orthogonal projection of $V$ onto $\tilde{V}$ is $P(\cdot)=\sum_{i=d+1}^{N} \tilde{x}_{i}^{\prime}(\cdot) x_{i}$.

Proof. By Proposition 3, $P(w)=w$ for all $w \in \tilde{V}$ and Range $(P)=\tilde{V}$. From Propositions 1 and 3, $\tilde{V}^{\prime}$ is the null space of $P$, and $\operatorname{Range}(P)$ and $\tilde{V}^{\prime}=\operatorname{Null}(P)$ are orthogonal, so $P$ is an orthogonal projection. 
The following generalizes [3, Corollary 3.2] to give the coefficients of $P(f)$ for the case $d \geq 1$.

Theorem 5. If $f=\sum_{i=1}^{N} c_{i} x_{i}$, where $c_{i}=\left\langle x_{i}^{\prime}, f\right\rangle$, then

$$
P(f)=\sum_{i=d+1}^{N} c_{i}^{\prime} x_{i}, \quad \text { where } c_{i}^{\prime}=c_{i}-\sum_{\ell=1}^{d} \frac{\left\langle x_{i}^{\prime}, v_{\ell}^{\prime}\right\rangle}{\left\langle v_{\ell}^{\prime}, v_{\ell}^{\prime}\right\rangle}\left\langle v_{\ell}^{\prime}, f\right\rangle .
$$

Proof. We calculate, using (2),

$$
P(f)=\sum_{i=d+1}^{N} \tilde{x}_{i}^{\prime}(f) x_{i}=\sum_{i=d+1}^{N}\left(\left\langle x_{i}^{\prime}, f\right\rangle-\sum_{\ell=1}^{d} \frac{\left\langle x_{i}^{\prime}, v_{\ell}^{\prime}\right\rangle}{\left\langle v_{\ell}^{\prime}, v_{\ell}^{\prime}\right\rangle}\left\langle v_{\ell}^{\prime}, f\right\rangle\right) x_{i} .
$$

so $P(f)=\sum_{i=d+1}^{N} c_{i}^{\prime} x_{i}$, where

$$
c_{i}^{\prime}=c_{i}-\sum_{\ell=1}^{d} \frac{\left\langle x_{i}^{\prime}, v_{\ell}^{\prime}\right\rangle}{\left\langle v_{\ell}^{\prime}, v_{\ell}^{\prime}\right\rangle}\left\langle v_{\ell}^{\prime}, f\right\rangle .
$$

The following generalizes [3, Corollary 3.3] for the case $d \geq 1$.

Corollary 6. If $f=\sum_{i=1}^{N} c_{i} x_{i}$, where $c_{i}=\left\langle x_{i}^{\prime}, f\right\rangle$, then

$$
\|f\|^{2}=\|P(f)\|^{2}+\sum_{i=1}^{d} \frac{1}{\left\|v_{i}^{\prime}\right\|^{2}}\left|\sum_{k=1}^{i} c_{k}\left\langle v_{i}^{\prime}, x_{k}\right\rangle\right|^{2} .
$$

Proof. Since $V=\tilde{V} \oplus \tilde{V}^{\prime}$, we can write $f=P(f) \oplus \operatorname{proj}_{\tilde{V}^{\prime}}(f)$, where $\operatorname{proj}_{\tilde{V}^{\prime}}(f)=$ $\sum_{i=1}^{d}\left\langle v_{i}^{\prime} /\left\|v_{i}^{\prime}\right\|, f\right\rangle\left(v_{i}^{\prime} /\left\|v_{i}^{\prime}\right\|\right)$ is the projection of $f$ onto $\tilde{V}^{\prime}$ using the orthogonal basis $\left\{v_{i}^{\prime}\right\}$. Thus by Parseval and then Lemma 2, we have

$$
\begin{aligned}
\|f\|^{2} & =\|P(f)\|^{2}+\left\|\sum_{i=1}^{d}\left\langle\frac{v_{i}^{\prime}}{\left\|v_{i}^{\prime}\right\|}, f\right\rangle \frac{v_{i}^{\prime}}{\left\|v_{i}^{\prime}\right\|}\right\|^{2}=\|P(f)\|^{2}+\sum_{i=1}^{d} \frac{1}{\left\|v_{i}^{\prime}\right\|^{2}}\left|\left\langle v_{i}^{\prime}, f\right\rangle\right|^{2} \\
& =\|P(f)\|^{2}+\sum_{i=1}^{d} \frac{1}{\left\|v_{i}^{\prime}\right\|^{2}}\left|\sum_{k=1}^{i} c_{k}\left\langle v_{i}, x_{k}\right\rangle\right|^{2} .
\end{aligned}
$$

Next we generalize [3, Proposition 3.4] for the case $d \geq 1$.

Proposition 7. By reindexing the original $x_{i}$ and $x_{i}^{\prime}$ to examine all possible $\left(\begin{array}{l}N \\ d\end{array}\right)$ combinations of $d$ components dropped from the original basis of $V$ and to minimize the $L^{2}$ distance between $f$ and $P(f)$, choose the set of $d$ basis elements $x_{i}$ that minimizes

$$
\sum_{i=1}^{d} \frac{1}{\left\|v_{i}^{\prime}\right\|^{2}}\left|\sum_{k=1}^{i} c_{k}\left\langle v_{i}^{\prime}, x_{k}\right\rangle\right|^{2}
$$

We now give an example demonstrating that iterating the process $k$ times with $d=1$ may give a projection considerably farther from the original $f$ than reducing by $k=d$ basis functions simultaneously. 


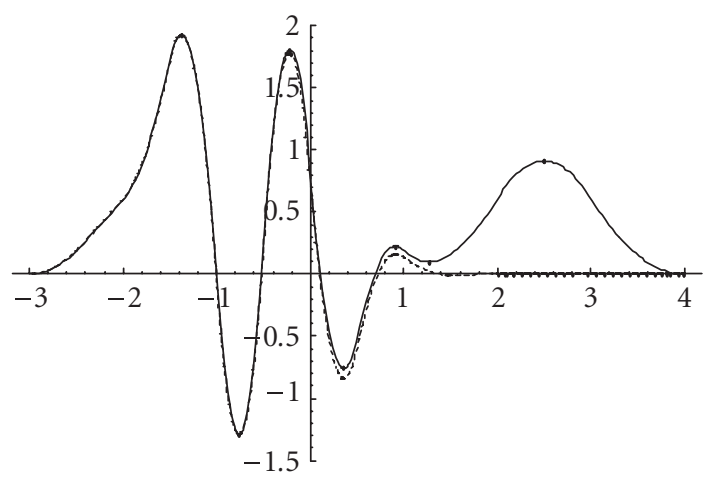

(a)

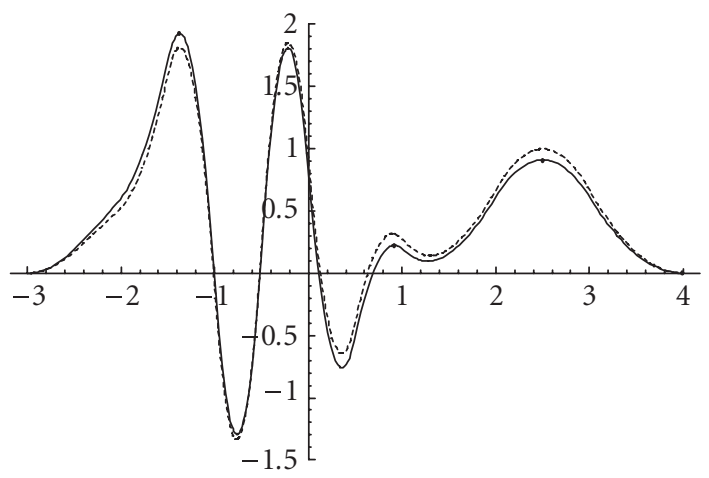

(b)

Figure 1. Drop two basis functions: iteratively (a), and simultaneously (b), for Example 8.

Example 8. For simplicity, we consider a function $f(t)$ in the four-dimensional subspace $V$ with basis functions generated from cardinal spline wavelets. Let $B_{3}(x)$ be the standard quadratic cardinal spline supported on $[-1,2]$ and let $w(t)$ be the standard associated wavelet for the Riesz basis of $L^{2}(\mathbb{R})$ generated by $B_{3}(x)$ as mentioned in [1] or [2]. Let $V=\operatorname{span}\left\{x_{1}, x_{2}, x_{3}, x_{4}\right\}$, where $x_{1}(t)=B_{3}(t+2) /\left\|B_{3}\right\|, x_{2}(t)=B_{3}(t-2) /\left\|B_{3}\right\|, x_{3}(t)=$ $\left(B_{3}(t-2)+B_{3}(t+2)+0.2 B_{3}(t)\right) /\left\|B_{3}\right\|, x_{4}=w(t)$. The function $f$ can be expressed as $f(t)=0.7 x_{1}(t)+0.5 x_{2}(t)+0.4 x_{3}(t)+x_{4}(t)$. We wish to drop $d=2$ basis elements and obtain the best two-dimensional approximation to $f$. If we iteratively drop one basis element at a time using Proposition 7 with $d=1$, then we remove $x_{3}$ and then $x_{2}$ leaving projection $P(f)=0.9 x_{1}+x_{4}$ as shown in Figure $1(\mathrm{a})$ with residual error $\|f-P(f)\|^{2}=0.82$. However, if we simultaneously drop two elements with $d=2$, then we instead drop $x_{1}$ and $x_{2}$ leaving projection $P(f)=1.1 x_{3}+x_{4}$ as shown in Figure 1(b) with residual error $\|f-P(f)\|^{2}=0.03$. As can be seen from these errors and the plots in Figure 1, there is a considerable advantage for $t \geq 1.5$ in removing two basis elements together, rather than dropping them iteratively. 
When the value of $\left(\begin{array}{l}N \\ d\end{array}\right)$ is large, the computational expense of choosing the optimal set of basis elements to be dropped can be quite large. Investigation of this issue merits further study.

\section{References}

[1] I. Daubechies, Ten Lectures on Wavelets, CBMS-NSF Regional Conference Series in Applied Mathematics, vol. 61, SIAM, Pennsylvania, 1992.

[2] P. R. Massopust, D. K. Ruch, and P. J. Van Fleet, On the support properties of scaling vectors, Applied and Computational Harmonic Analysis 3 (1996), no. 3, 229-238.

[3] L. Rebollo-Neira, Backward adaptive biorthogonalization, International Journal of Mathematics and Mathematical Sciences 2004 (2004), no. 35, 1843-1853.

David K. Ruch: Department of Mathematical and Computer Sciences, Metropolitan State College of Denver, Denver, CO 80217-3362, USA

E-mail address: ruch@mscd.edu 


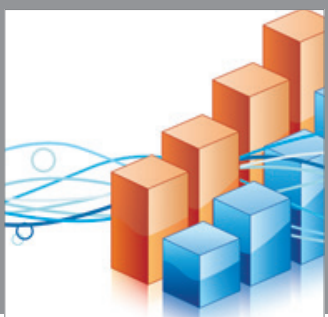

Advances in

Operations Research

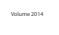

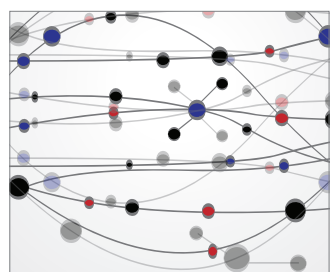

\section{The Scientific} World Journal
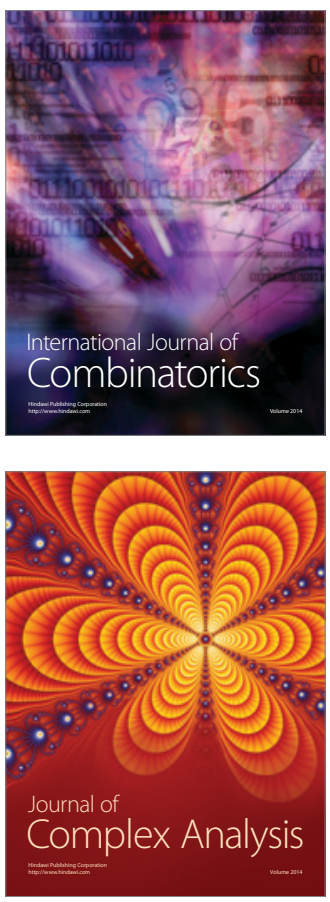

International Journal of

Mathematics and

Mathematical

Sciences
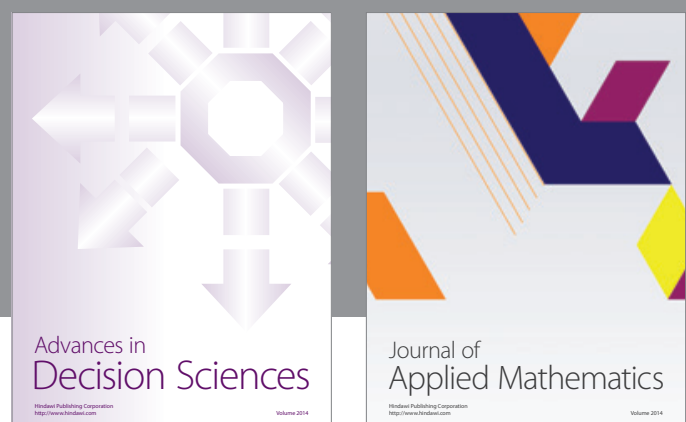

Journal of

Applied Mathematics
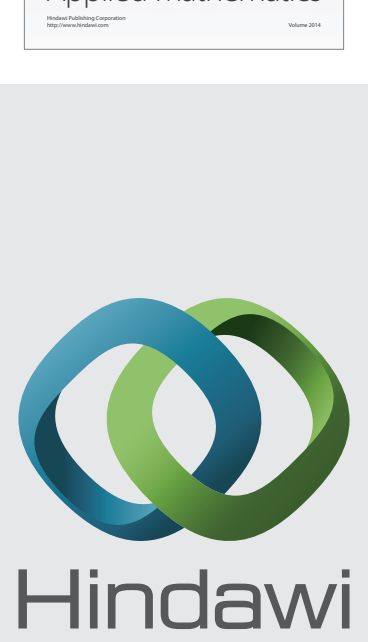

Submit your manuscripts at http://www.hindawi.com
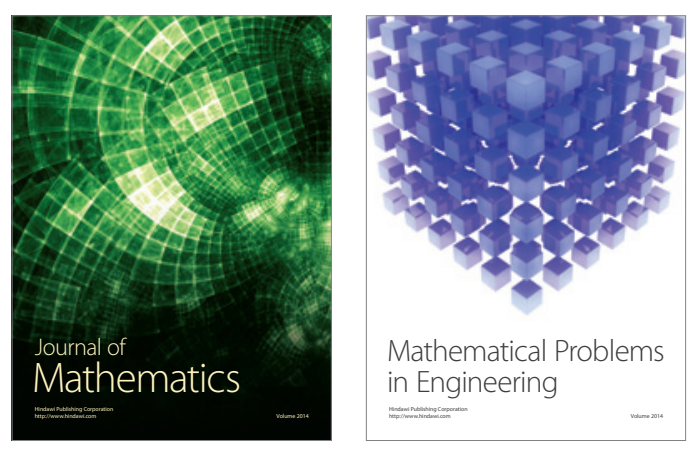

Mathematical Problems in Engineering
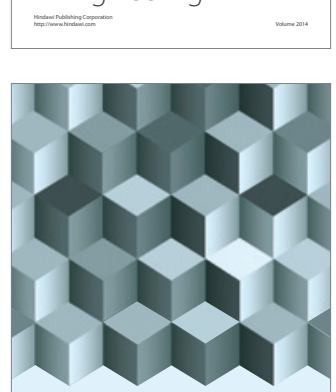

Journal of

Function Spaces
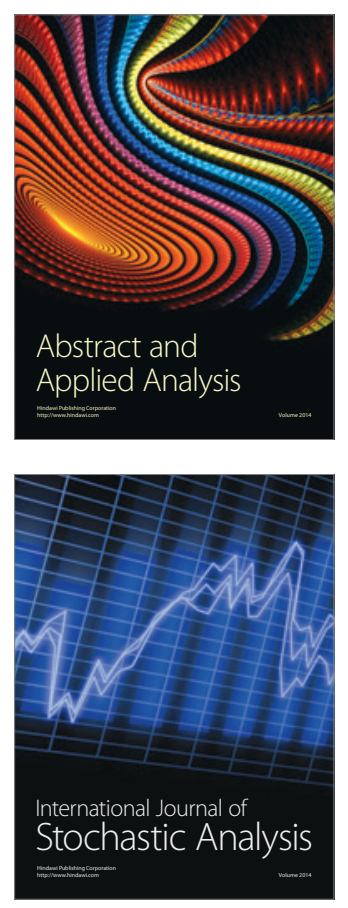

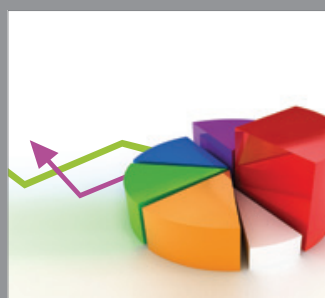

ournal of

Probability and Statistics

Promensencen
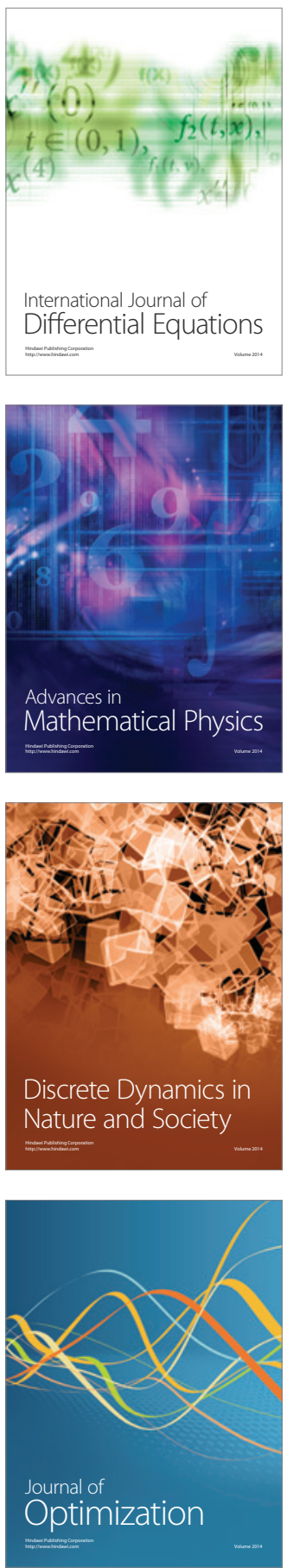